\title{
Maximum-likelihood psychometric procedures in two-alternative forced-choice: Evaluation and recommendations
}

\author{
ROBERT MADIGAN and DAVID WILLIAMS \\ University of Alaska, Anchorage, Anchorage, Alaska
}

\begin{abstract}
Several new adaptive psychometric procedures have been proposed that use maximum-likelihood methods to estimate points on a subject's psychophysical function. These developments are summarized, and some psychometric situations that pose special problems for the new approaches are examined. The problem areas include the effects of threshold changes during the testing session, the impact of errors made by the subject, and differences between yes-no and two-alternative forced-choice situations. Data are presented from both human subjects and computer simulations. Strengths and weaknesses of the new procedures are identified and recommendations are made for their use.
\end{abstract}

Adaptive psychometric procedures estimate points on the psychophysical function by making use of the subject's previous responses to select new stimuli for testing. Recently, there have been several proposals to use maximum-likelihood estimation in conjunction with adaptive testing (Hall, 1981; Pentland, 1980; Watson \& Pelli, 1983). Data presented in these papers suggest that this approach represents an important advance in the sophistication of psychometric measurement. The present paper reviews these developments. Computer simulations are used to examine some psychometric situations that pose special problems for the new approaches, with a special emphasis on the two-alternative forced-choice (2AFC) paradigm. A human-subjects experiment is also reported in which thresholds estimated with two of the maximumlikelihood procedures were compared with thresholds obtained using one of the established adaptive procedures. The paper concludes with recommendations for the selection of adaptive procedures.

\section{MAXIMUM-LIKELIHOOD METHODS}

The use of maximum-likelihood methods as part of an adaptive psychometric procedure can be illustrated by Pentland's (1980) approach, named the Best PEST. The procedure is designed to estimate the stimulus at the midpoint of the psychometric curve, which is a stimulus identified on $50 \%$ of presentations in a yes-no task or on $75 \%$ of presentations in $2 \mathrm{AFC}$. For convenience, this stimulus is called the threshold.

A portion of this work was presented at the annual meeting of the American Psychological Association, Washington, D.C., August, 1986. We gratefully acknowledge Sharon Ringwelski's assistance in the data collection and Bernie Segal's comments on a draft of this article. Requests for reprints should be sent to Robert Madigan, Department of Psychology, University of Alaska, Anchorage. Anchorage, AK 99508.
The Best PEST procedure assumes that the subject's psychometric function is described by a logistic curve (Guilford, 1954):

$$
P(\text { Correct } \mid S)=g+(1-g) /\left(1+e^{(T-A) / B}\right),
$$

where $S$ is a measure of stimulus intensity, $T$ is a threshold parameter, $B$ is the slope parameter, and $g$ is the guessing probability, which is assumed to be 0 for yes-no and .5 for $2 \mathrm{AFC}$. The purpose of Pentland's procedure is to estimate the threshold parameter of this function. A reasonable guess is made about the value of the slope parameter and a set of possible threshold estimates is established based on past experience. The test stimuli are drawn from this set. The likelihood that each stimulus in the set of test stimuli is the subject's threshold is evaluated after every response. This is accomplished by calculating the probability of the subject's observed responses to all past stimuli, given that the logistic threshold parameter has a particular value. The stimulus value with the maximum likelihood is presented as the test stimulus on the next trial. After a predetermined number of trials, the procedure ends with the final maximum-likelihood stimulus as the threshold estimate.

The three maximum-likelihood techniques differ in a number of important details, which are summarized in Table 1.

\section{The Psychometric Curve}

All three maximum-likelihood proposals are based on an assumed psychometric curve that is used to calculate the likelihood statistics. Both Hall (1981) and Pentland (1980) use the logistic function to represent the subject's psychometric function. Hall formally estimates both the threshold and the slope parameter for each subject, whereas Pentland estimates only the threshold and uses a fixed estimate of the slope parameter for all subjects.

Watson and Pelli (1983) employ the Weibull psychometric function (Green \& Luce, 1975) to calculate likeli- 
Table 1

Comparisons of Three Maximum-Likelihood Procedures

\begin{tabular}{|c|c|c|c|}
\hline & Hall (1981) & Pentland (1980) & $\begin{array}{l}\text { Watson \& } \\
\text { Pelli (1983) }\end{array}$ \\
\hline $\begin{array}{l}\text { Assumed psychometric } \\
\text { function }\end{array}$ & Logistic & Logistic & Weibull \\
\hline $\begin{array}{l}\text { Parameters estimated } \\
\text { for each subject }\end{array}$ & $\begin{array}{l}\text { Threshold, } \\
\text { slope }\end{array}$ & Threshold & Threshold \\
\hline \multicolumn{4}{|l|}{$\begin{array}{l}\text { Probability of } \\
\text { detection at } \\
\text { threshold parameter }\end{array}$} \\
\hline $\begin{array}{l}\text { Yes-No } \\
\text { 2AFC }\end{array}$ & $\begin{array}{l}.50 \\
.75\end{array}$ & $\begin{array}{l}.50 \\
.75\end{array}$ & $\begin{array}{l}.80 \\
.92\end{array}$ \\
\hline $\begin{array}{l}\text { Test stimulus } \\
\text { spacing }\end{array}$ & $\begin{array}{l}\text { Not } \\
\text { critical }\end{array}$ & $\begin{array}{l}\text { Equal } \\
\text { psychometric } \\
\text { units }\end{array}$ & $\begin{array}{l}\text { Equal } \\
\text { psychometric } \\
\text { units with } \\
\text { stimuli } \\
\text { logarithmically } \\
\text { scaled }\end{array}$ \\
\hline $\begin{array}{l}\text { Selection of } \\
\text { stimuli during } \\
\text { testing }\end{array}$ & $\begin{array}{l}\text { Taylor \& } \\
\text { Creelman's (1967) } \\
\text { PEST }\end{array}$ & $\begin{array}{l}\text { Maximum- } \\
\text { likelihood } \\
\text { threshold }\end{array}$ & $\begin{array}{l}\text { Maximum- } \\
\text { likelihood } \\
\text { threshold } \\
\text { including } \\
\text { a priori } \\
\text { information }\end{array}$ \\
\hline Stopping rule & $\begin{array}{l}\text { Taylor \& } \\
\text { Creelman's (1967) } \\
\text { PEST rules }\end{array}$ & $\begin{array}{l}\text { Fixed } \\
\text { trials }\end{array}$ & $\begin{array}{l}\text { Likelihood } \\
\text { ratio }\end{array}$ \\
\hline Final estimate & $\begin{array}{l}\text { Maximum } \\
\text { likelihood }\end{array}$ & $\begin{array}{l}\text { Maximum } \\
\text { likelihood }\end{array}$ & $\begin{array}{l}\text { Maximum } \\
\text { likelihood } \\
\text { excluding } \\
\text { a priori } \\
\text { information }\end{array}$ \\
\hline
\end{tabular}

hood values in their QUEST procedure. It is assumed that stimuli have been scaled in a form

$$
S=c \log I+K,
$$

where $I$ is a measurement of the physical intensity of the stimulus and $c$ and $K$ are constants. Under this condition, the Weibull has the form

$$
(P \mid S)=1-(1-g) e^{-10^{B(S-T) / C}} \text {. }
$$

$T$ and $B$ are the threshold and slope parameters, respectively, and the other symbols are as defined above. The threshold parameter of the Weibull is a stimulus value that has a yes-no detection probability of .62 , rather than .50 as in the logistic function. Watson and Pelli use the same estimate of the Weibull slope parameter for all subjects. The parameter $g$ is assumed to be 0 for yes-no and .5 for $2 \mathrm{AFC}$.

It is possible to estimate points other than the threshold parameter of the assumed psychometric function by replacing $T$ in Equation 3 by

$$
T=T^{\prime}+d
$$

where $T^{\prime}$ is the stimulus corresponding to the point of interest and $d$ is a fixed offset equal to the difference between $T$ and $T^{\prime}$. The parameter $d$ is calculated using the Weibull assumed slope parameter. When this transforma- tion is used, the procedure then presents all test stimuli at the best estimate of $T^{\prime}$. Watson and Pelli (1983) recommend this approach for $2 \mathrm{AFC}$ to estimate the stimulus with a detection probability of .92 , which can be shown to be a particularly efficient testing point.

\section{The Stimulus Set}

Both Pentland (1980) and Watson and Pelli (1983) calculate maximum-likelihood statistics on every trial. The computations are made manageable by a judicious selection of test stimuli. Pentland selects stimuli that are at equal intervals along the abscissa of the psychometric curve. Because of the nature of the Weibull function, Watson and Pelli's approach requires that the stimuli be at equal logarithmic units on a scale of physical intensity. This means that the Weibull function requires that the stimuli satisfy Weber's law, but the logistic function used by Pentland does not.

Hall (1981) calculates likelihood statistics only after the testing is complete, and this relaxes the requirement that test stimuli be selected at equal intervals for rapid computation.

\section{Selecting the Testing Levels}

Table 1 shows differences among the approaches in the way the next testing level is selected. Hall (1981) uses 
the well-established PEST procedure (Taylor \& Creelman, 1967), with some refinements. This is an up-down approach in which the amount of stimulus change is reduced as the subject's threshold is bracketed. Testing is terminated when the size of the stimulus change reaches a predetermined minimum level. After the testing has been concluded, Hall uses the maximum-likelihood procedure with the subject's response sequence to estimate the threshold.

Both Pentland (1980) and Watson and Pelli (1983) employ the maximum-likelihood method to select the next test stimulus so that all testing is done at the best available estimate of the subject's threshold. The important difference between their approaches is that Watson and Pelli's calculations include a priori information about the probability density function of the threshold and Pentland's do not.

\section{Stopping Rules}

Hall (1981) terminates testing according to modified PEST criteria in which the number of trials depends on the subject's performance. The procedures proposed by Pentland (1980) and Watson and Pelli (1983) give the experimenter options for how the testing should be ended. One strategy is to run a predetermined number of trials, and this is the approach recommended by Pentland. Watson and Pelli develop an alternative in which the likelihood values are used to construct a confidence interval around the threshold. When the confidence interval meets a narrowness criterion, the testing is terminated.

\section{EFFICIENCY}

Some data on the efficiency of maximum-likelihood procedures are available from Monte Carlo simulations and from experiments with human subjects. Pentland (1980) presents computer simulations of his procedure in a yes-no task and compares the variability of threshold estimates to other simulation data reported by Findlay (1978), which included a simple staircase (Cornsweet, 1962) and the PEST procedure of Taylor and Creelman (1967). In Pentland's simulations, the maximumlikelihood procedure is clearly superior to the others.

The relative efficiency of the maximum-likelihood approach in computer-simulated 2AFC has not been reported. Shelton, Picardi, and Green (1982), using data from human subjects, compared a staircase method, Taylor and Creelman's (1967) PEST procedure, and Pentland's (1980) approach. Only small differences among the approaches emerged form their study. This result was surprising in light of the dramatic differences between the procedures in simulated yes-no data.

We conducted computer simulations of Pentland's (1980) Best PEST and Watson and Pelli's (1983) QUEST procedures in 2AFC and yes-no situations. The purpose of the simulations was to compare the two maximumlikelihood procedures directly and to provide simulation data on the behavior of maximum-likelihood methods in the common $2 \mathrm{AFC}$ situation. The findings will also serve as a baseline for evaluating the behavior of the procedures under the less than optimal conditions investigated later.

No results are reported for Hall's (1981) procedure because it does not appear suitable for 2AFC. Hall developed his approach for four-alternative forced-choice (4AFC) and reported that it is inefficient for 2AFC.

Simulations are also reported for an alternative to maximum-likelihood methods, Taylor and Creelman's (1967) PEST procedure, one of the best-known and mostoften studied of the adaptive methods.

\section{Simulation Protocol}

The simulations of yes-no tasks followed recent work (Emerson, 1984; Findlay, 1978; Pentland, 1980) in assuming that the probability of correctly detecting the stimulus varies from 0 to 1 according to a psychometric curve. The subject never guesses. The subject's true psychometric curve is a logistic function for simulations of Pentland's (1980) Best PEST and Taylor and Creelman's (1967) PEST. The subject is assumed to have a Weibull psychometric function in simulations of QUEST. All simulated subjects have psychometric curves with the same slope.

The use of two psychometric functions creates a problem of comparable units, because stimuli are scaled in logit units for two of the procedures and in different units for the Weibull function. Logits were selected as the units of analysis for this paper. The slope parameter of the logistic curve describing the subject's true psychometric function is taken as 1 logit unit. In a yes-no task, 4.6 logit units cover the range between a stimulus detected with probability 0.50 and one detected $99 \%$ of the time. A stimulus 1 logit unit above threshold in a yes-no task is detected with probability .73 . Weibull units were converted to logit units by dividing them by 1.71 , a value found by using a least squares approach to fitting the Weibull function to the logistic when the Weibull slope and stimulus scaling parameters were set to the values suggested by Watson and Pelli (1983) $(B=3.5, c=20)$.

The test stimuli in the simulations were spaced .5 logit units apart over a range of 8 logits. The subject's true threshold was randomly selected to be a value within \pm 3 logit units of the middle of the stimulus set. One hundred subjects were simulated for each condition reported.

2AFC was simulated by assuming that subjects guess with probability .5 whenever they fail to detect the stimulus. All procedures estimated the point on the psychometric curve detected with probability .50 in yes-no and .75 in $2 \mathrm{AFC}$.

\section{Implementation of Maximum-Likelihood Procedures}

Pentland's procedure was implemented following the computer program presented by Lieberman and Pentland 
(1982). The procedure was terminated after a fixed number of judgments. Watson and Pelli's (1983) QUEST procedure was implemented to run until a confidence interval for the threshold was smaller than a specified size. Preliminary work with the procedure suggested that a confidence interval of \pm 2 logit units worked reasonably well for 2AFC. Varying the level of confidence between $90 \%$ and $99.5 \%$ caused the procedure to run an average of between 25 and 60 trials. For yes-no, a confidence interval of \pm 1 logit was used and the confidence level was varied as needed to obtain runs of between 10 and 50 trials.

The QUEST procedure requires the experimenter to specify an a priori distribution of threshold estimates. The simulations used the a priori distribution suggested by Watson and Pelli (1983) (with a standard deviation of 12 Weibull units) centered over the stimuli from which the threshold was randomly selected.

\section{Implementation of PEST}

In Taylor and Creelman's (1967) PEST procedure, the experimenter establishes a testing level and presents stimuli for judgment. A Wald test (Wald, 1947) is used to decide when to change levels by comparing the number of correct responses observed at the current testing level with the number expected if the testing stimulus were at the desired point on the psychometric curve. When this difference equals or exceeds a criterion, the testing level is adjusted up or down. If the adjustment is in the opposite direction from the last testing level adjustment, in- dicating that the desired point is being bracketed, the size of the adjustment is halved. When the adjustment is in the same direction as the preceding one, the amount of stimulus adjustment is doubled if (1) it is the fourth or more step in the same direction or (2) it is the third step in the same direction and the last occurrence of three adjustments in the same direction did not lead to a doubling of the step size. The Taylor and Creelman procedure terminates when the adjustment size reaches a predetermined value. For the data reported here, we used a final step size of between .1 and 1.0 logit units. It should be pointed out that a fixed-trials adaptation of the PEST procedure has been reported (Creelman \& Kaplan, 1973), but it has received little attention. We used the variable-trials version in this study.

\section{Simulation Results}

Figure 1 presents the simulation data. The setting accuracy statistic is the standard deviation of the threshold estimation errors. The data simulating yes-no judgments show smaller error variances for the maximum-likelihood approaches than for Taylor and Creelman's PEST. It appears from Figure 1 that there is no difference between the variability of threshold estimates produced by the QUEST procedure and the variability of the estimates produced by Pentland's Best PEST.

The 2AFC simulations show a similar pattern. The two maximum-likelihood procedures differ little and both are consistently superior to Taylor and Creelman's PEST.

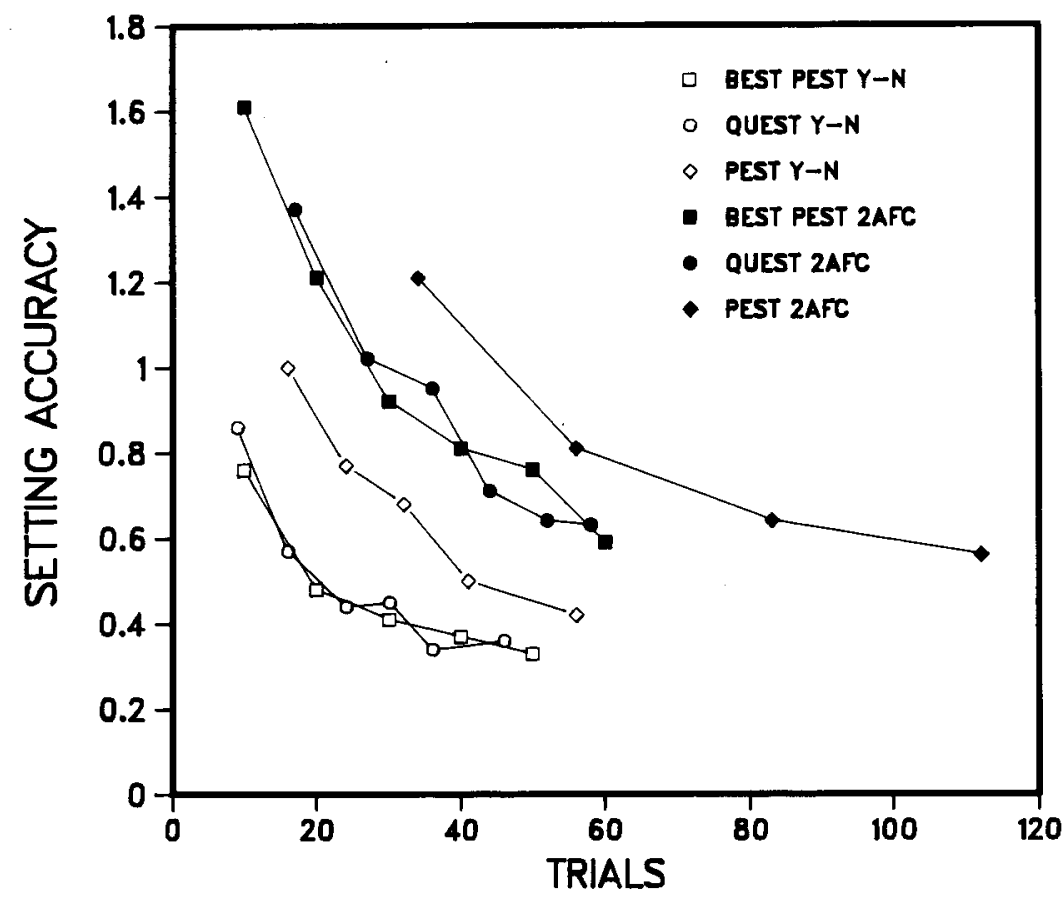

Figure 1. A comparison between maximum-likelihood psychometric procedures and Taylor and Creelman's (1967) PEST approach. Setting accuracy is the standard deviation of threshold estimation errors. $\mathrm{Y}-\mathrm{N}=$ yes-no; $\mathbf{2 A F C}=$ two-alternative forcedchoice. 
It is clear that $2 A F C$ is less efficient than the yes-no paradigm simulated in the figure. The reason for this is the high guessing probability in 2AFC. In fact, 2AFC, despite its many advantages, is inherently more variable than any other common testing format in terms of its .50 guessing probability (McKee, Klein, \& Teller, 1985). In $4 \mathrm{AFC}$, the guessing probability drops to .25 , which considerably reduces the variability of the procedure and may be the reason that Hall's procedure can be demonstrably successful in 4AFC yet not do well in 2AFC (Hall, 1981).

The 2AFC data of Figure 1 do not predict Shelton et al.'s (1982) failure to find significant differences between PEST and Pentland's (1980) Best PEST. However, the absolute differences between the procedures in the figure are not great. The data suggest that maximumlikelihood procedures are affected by the guessing-induced variability in $2 \mathrm{AFC}$, and this raises questions about their performance in situations where other sources of variability are operating. Some of these are explored below.

\section{SPECIAL SITUATIONS}

There are several factors that may adversely affect the efficiency of the maximum-likelihood procedures, such as subjects' learning to discriminate the stimulus better during the testing session, or subjects' occasionally lapsing in their performance. This section explores the effects of some of these factors on the behavior of adaptive procedures in simulated 2AFC tasks.

Nonstationary performance in the form of learning or performance lapses is a major challenge for any psychophysical procedure, but it poses special problems for maximum-likelihood approaches because the entire response sequence is used to estimate the threshold. Taylor, Forbes, and Creelman (1983) suggested that this is a major flaw in the maximum-likelihood approach. Adaptive procedures such as Taylor and Creelman's (1967) PEST seem less vulnerable to biasing from the effects of nonstationary performance because they rely only on the later trials of the sequence for the final threshold estimate.

\section{Learning}

Frequently, psychophysical procedures are used to measure thresholds of inexperienced subjects. In these situations, it may be expected that the threshold is decreasing during the measurement session, an effect reported by a several investigators (Hall, 1983; Wetherill \& Levitt, 1965).

The simulated effects of learning in $2 \mathrm{AFC}$ were investigated for the maximum-likelihood procedures and Taylor and Creelman's (1967) PEST. An acquisition curve must be assumed in order to simulate learning, and it appears that little is known about the shape of this function. We arbitrarily selected for the simulation runs a simple linear acquisition curve that assumed that learning took place in the first 20 trials of testing. During this time, the subject's true threshold was decreased by a fixed amount on each trial until it reached its final value on
Trial 21. The total threshold change was assumed to be 2 logit units.

Figure 2 summarizes the results of these simulations in terms of the mean threshold estimation error as a function of the number of trials. It is important to note that both maximum-likelihood procedures underestimate the threshold in the absence of learning, especially when the run length is short. Learning effects are superimposed on this bias and move from an underestimate of the threshold to an overestimate. All procedures are impressive in their ability to tolerate a significant amount of simulated learning in the early trials of a session yet yield reasonable threshold estimates by 60 trials.

\section{Lapses}

It has long been known that the performance of subjects making psychophysical judgments is not stationary but varies during the testing run, even after extensive experience (Shipley, 1961). The nature of these changes is not well understood. There are many potential sources of lapses in performance levels, including errors, motivational factors, and sensory changes. Taylor et al. (1983)

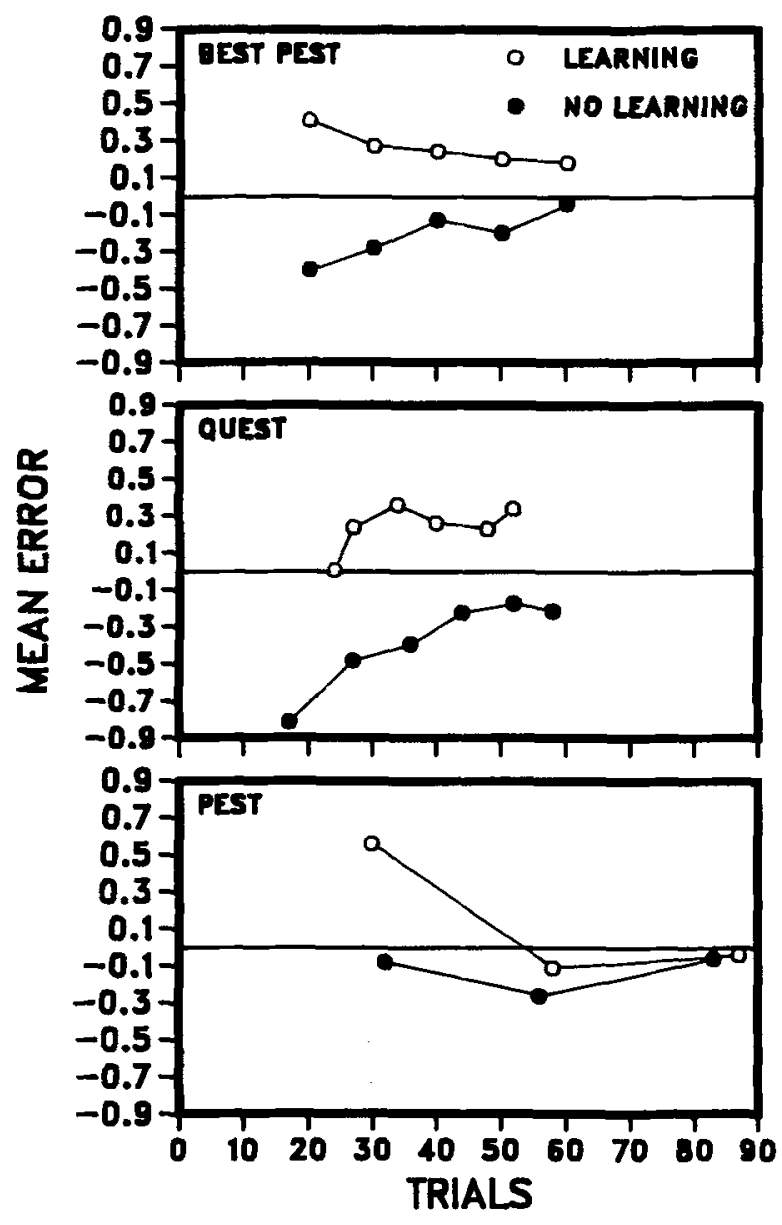

Figure 2. The effects of within-session threshold changes on the accuracy of threshold estimation by three procedures. Thresholds decreased by 2 logit units over the first 20 trials. 
suggested that actual performance levels may vary anywhere from chance to periods of high sensitivity at different points in the session. Shipley's (1961) data suggest that there are individual differences in the extent to which these factors affect different subjects.

2AFC simulations of the maximum-likelihood procedures and the original PEST were performed with lapse probabilities of 0 and .2 . The parameters of each method were adjusted to give run lengths of about 40 trials. A lapse was defined analogously to not attending to the stimulus: On a lapse trial, the detection probability dropped to the guessing level.

The susceptibility of the maximum-likelihood methods and the original PEST procedure to lapses in a 2AFC situation is explored in Figure 3. The data are presented as setting accuracies. The figure shows that the Best PEST is most affected by lapses, although the absolute differences among the approaches are small and are unlikely to have practical significance. Lapses also affect the point on the psychometric curve that is estimated by the procedures. As lapsing increases, the estimated point is farther from the true threshold. At a 20\% lapse rate, for example, the threshold was estimated to be .55 logit units above the true threshold. This corresponds to a stimulus with a 2 AFC detection probability of .82 under no lapsing conditions.

\section{Instabilities in the Best PEST}

In the simulations above, the stimulus range was held to 8 logits. Ranges of 32,16 , and 8 logit units were simulated to explore their effects on the maximum-likelihood procedures; these ranges revealed Pentland's (1980) procedure to be sensitive to the range of stimuli being tested. Emerson (1986) reported a similar finding.

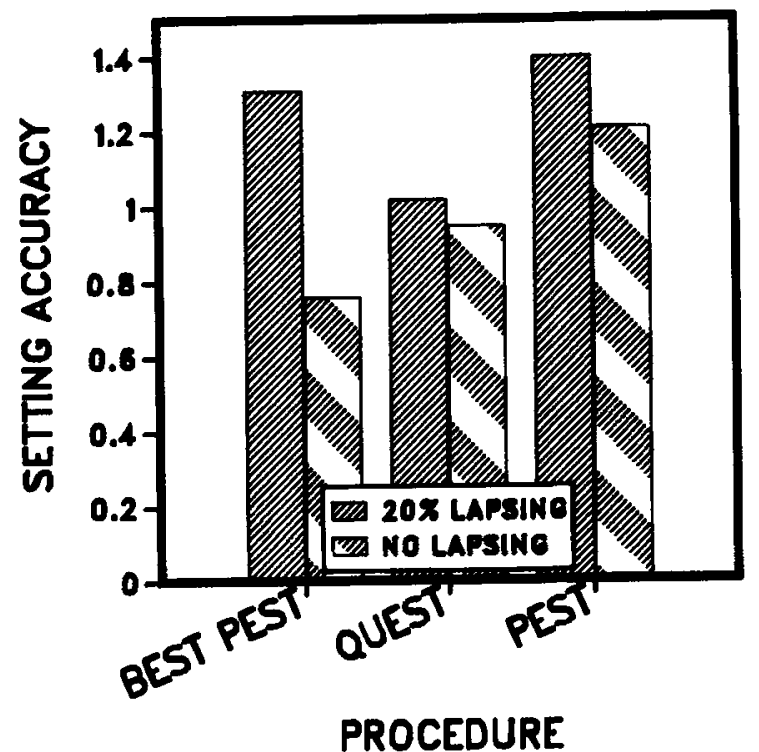

Figure 3. The effects of performance lapses on the setting accuracy of three adaptive procedures.

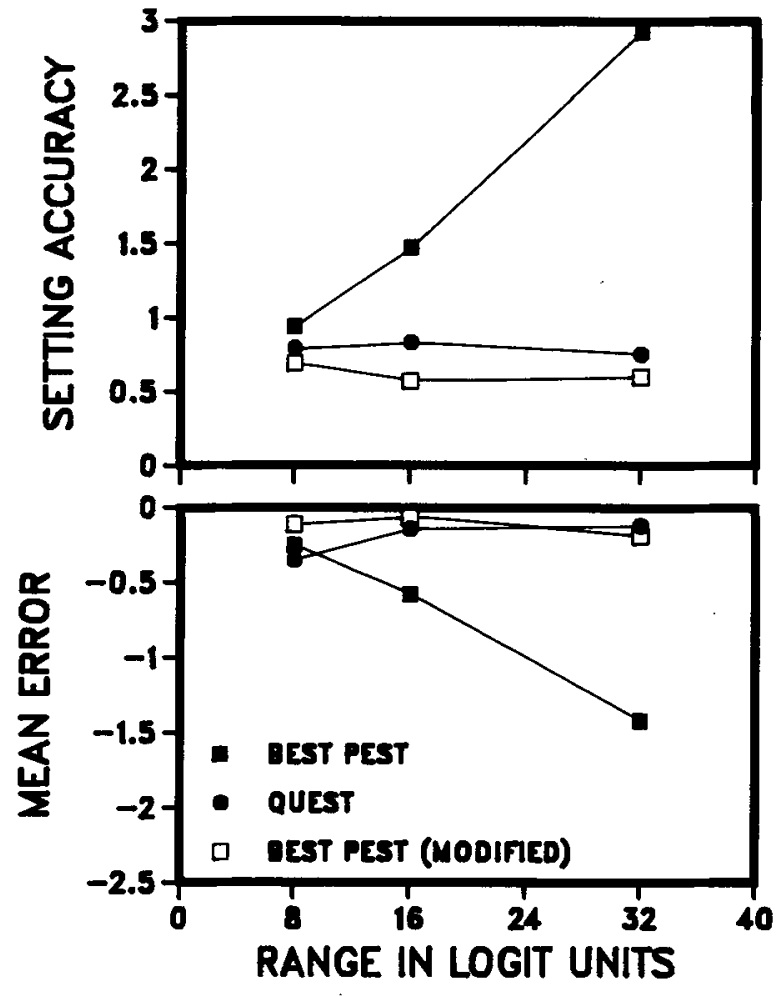

Figure 4. The effects of the range of test stimuli on the setting accuracy of three psychophysical procedures. The modified Best PEST procedure incorporates a priori information about the distribution of threshold values.

Figure 4 presents simulation data that show that increasing the range of stimuli causes an increase in both the variability as measured by the setting accuracy and the absolute mean error of the Best PEST threshold estimate. As the range becomes greater, the threshold is increasingly underestimated.

The QUEST procedure (Watson \& Pelli, 1983) is not affected by changes in the range. We attribute the difference between the performances of QUEST and the Best PEST to the a priori distribution used by QUEST. The a priori distribution is used to set the initial likelihood values for the test stimuli at the beginning of testing and is important for stimulus selection mainly during the early trials. This apparently constrains the selection of test stimuli in QUEST during the early trials and stabilizes the procedure. Stimulus selection in the Best PEST is constrained when the range is relatively narrow, but wider ranges allow the procedure occasionally to run amok, producing increased variability, as shown in Figure 4. Although not shown in the figure, lapsing can significantly increase this range-induced instability.

We tested a modified Best PEST in which the a priori distribution from the QUEST procedure was used to set the initial likelihood values for the stimuli. These simulations are also plotted in Figure 4. The modified Best PEST was as stable as QUEST, showing the importance of the a priori distribution. 
Watson and Pelli (1983) suggest a technique that could have effects on the stability of the PEST procedure. They propose truncating the psychometric function at some value (e.g., .95) as a way of dealing with lapsing. We wondered whether a truncated psychometric curve might dampen some of the wild swings in the Best PEST likelihood function that result from lucky guesses early in the session. We ran the Best PEST with the psychometric function truncated at values between .80 and .99 , but this manipulation failed to stabilize the procedure.

\section{Slope Estimation Errors}

The maximum-likelihood procedures require that the experimenter supply a value for the slope parameter of the assumed psychometric function. If the experimenter is incorrect in the choice of slope parameter, mismatches may occur between the slope parameter of the assumed psychometric function and the slope of the subject's true psychometric function. Mismatches may be expressed as the ratio of the true slope parameter to the assumed slope parameter.

Emerson (1984) showed that the Best PEST was largely insensitive to errors in the estimation of the slope parameter when used in the yes-no paradigm. Lieberman and Pentland (1982) suggested that it can be advantageous to overestimate the subject's slope parameter when lapsing is likely, and our own laboratory experience with the Best PEST supports this suggestion. Simulations were run to examine the effects of slope estimation errors in 2 AFC when lapsing is occurring. Mismatch ratios, defined as the assumed slope parameter divided by the true slope parameter, were set to $.5,1,2$, and 4 . Lapsing was assumed to occur on $20 \%$ of the trials. The Best PEST terminated after 40 trials and the QUEST criteria were set to give approximately $\mathbf{4 0}$ trial run lengths when the mismatch ratio was 1.0 .

Figure 5 shows the results of these simulations. It appears that Pentland's (1980) procedure performs somewhat better when the assumed slope parameter is an overestimate of the subject's true slope parameter, although the effect is not large. Slope estimation errors seem to affect QUEST's simulated accuracy in the opposite direction, with underestimation associated with better performance. However, this is likely to be an artifact of the confidence-interval stopping rule used with QUEST. The number of trials required to satisfy the confidence interval varied with the slope mismatch; underestimating the slope increased the run length and overestimation shortened it. A mismatch ratio of .5 required an average of 90 trials to reach the stopping criterion, whereas a mismatch ratio of 4 required only 16 trials. Since the setting accuracy is dependent on the number of trials, it is to be expected that those conditions that run the most trials would have the best setting accuracy. When QUEST is run as a fixed-trials procedure, the slope mismatch function is similar to that of the Best PEST.

Overall, it appears that slope errors are not a serious problem for maximum-likelihood methods in 2AFC, but

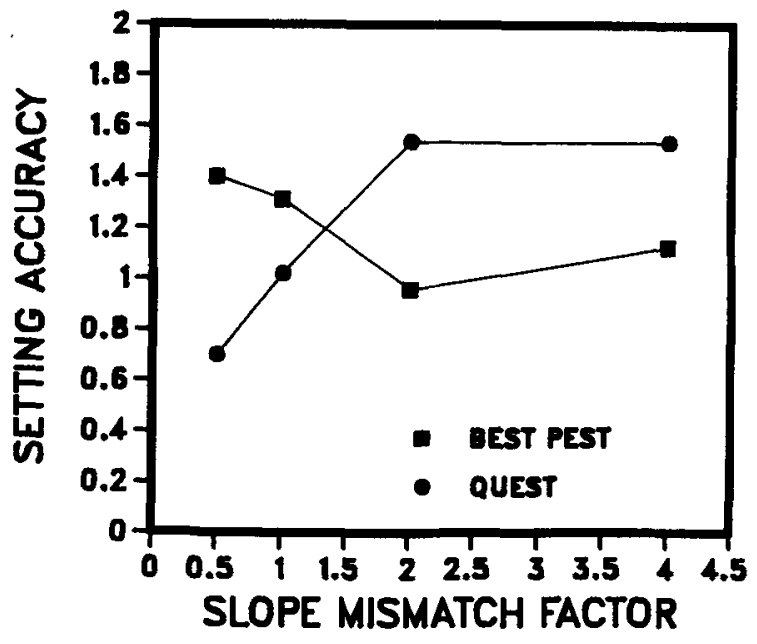

Figure 5. The effects of slope estimation errors on the setting accuracy of three psychophysical procedures. The mismatch ratio is the assumed slope parameter divided by the true slope parameter.

it is advantageous to overestimate the slope when using fixed trials, as in the Best PEST. Slope estimation errors in 2AFC do not affect QUEST's accuracy in the simulations, but errors do affect the number of trials required to reach the stopping criterion. Underestimating the slope parameter increases the run length, and overestimating shortens it. This points out an important characteristic of QUEST's confidence-interval approach: Not only is the run length affected by the confidence level selected by the investigator, it is also affected in an important way by the slope of the assumed psychometric function.

\section{HUMAN SUBJECTS}

An experiment was performed to compare directly threshold estimates obtained from the original PEST (Taylor \& Creelman, 1967), Pentland's (1980) Best PEST, and Watson and Pelli's (1983) QUEST. This experiment was motivated by several concerns. One reason for an empirical comparison was the lack of such data. Although simulations are useful for evaluating the sensitivity of procedures to specific situations, they are crude approximations to real human subjects in actual judgment situations. There have been two previous empirical comparisons between adaptive procedures. Shelton and Scarrow (1984) compared a simple adaptive staircase (Levitt, 1971) and Pentland's Best PEST, using inexperienced subjects who detected an auditory signal in noise. They found no differences between the procedures. Shelton et al. (1982) took repeated auditory threshold estimates from subjects, using three different procedures-the adaptive staircase method, Taylor and Creelman's PEST, and Pentland's maximum-likelihood approach. Each of 3 experienced subjects submitted to a total of 320 threshold determinations, and no differences were found between the methods.

The experiment presented below directly compared the two maximum-likelihood methods against the original 
PEST procedure in $2 \mathrm{AFC}$. Our study used inexperienced subjects. The novice must master a possibly unfamiliar sensory discrimination, using an unfamiliar response system in an unfamiliar setting. Both learning and response errors are likely. We were interested in whether differences would emerge among the original PEST, the Best PEST, and the QUEST procedures in this setting.

\section{Method}

Fifty-two college students, most of whom were recruited from an introductory psychology class, participated in the experiment. The subjects were presented with each procedure in a counterbalanced, repeated measures, Latin square design. They received a 5-min break between procedures.

A backward-masked 2AFC visual discrimination task was used in which stimuli were presented by computer-controlled projection tachistoscopes. The visual stimuli were a blank screen and an English word. Both stimuli were followed by a mask of jumbled letters. It was the subject's task to determine whether the first or second presentation contained the English word and to indicate a response by moving a lever. Auditory feedback was provided on every trial. The time between the presentation of the stimulus and the mask was adjusted to put the probability of a correct response at .75 .

The stimulus set. Data collected in a pilot study were used to select stimulus values and estimate parameters required for the QUEST and Best PEST procedures. The stimulus set consisted of 19 stimulus-mask durations in even logarithmic units from -8 to 10. The $\log$ units were derived from

$$
S=17 \log (D)-25,
$$

where $S$ is the logarithmic stimulus value and $D$ is an exposure duration in units peculiar to our computer system. The spacing of stimuli at the median threshold value was $1.6 \mathrm{msec}$. Pilot data suggested that this was aboutl logit unit for the typical subject.

Psychophysical procedures. Each session began with 2 demonstration trials that showed the stimuli and mask. These were followed by 10 practice trials in which the difficulty of the discrimination gradually increased from a level about 10 logit units above the typical subject's threshold to about 4 logit units above. At this point, the relevant psychometric procedure was started.

The Best PEST slope parameter was estimated from the data of 20 pilot subjects by a grid-search maximum-likelihood approach; the median slope was found to be 1.0 logarithmic units. Figure 5 suggests that overestimating the slope might help the effectiveness of the Best PEST in a situation where lapsing was likely, so the slope parameter used in the procedure was set to 2.0 logarithmic units. The Best PEST was programmed to run each subject for $\mathbf{5 0}$ trials.

The QUEST procedure used a Weibull slope parameter of $B=$ 5.6. This value was the median of the slope parameters estimated from the data of the pilot subjects, using the grid-search maximumlikelihood method. The Weibull parameter $c$ is determined from the stimulus scaling used and was 17 for our stimuli. In order to estimate the stimulus with a detection probability of .75 , Equation 4 must be used to replace $T$ in the Weibull function. The value calculated for $d$ was .48. The QUEST procedure also requires an a priori distribution of threshold likelihoods. In the QUEST procedure, the a priori distribution is used to set the log likelihood of the stimuli at the beginning of testing. These initial likelihood values were calculated using the approach suggested by Watson and Pelli (1983):

$$
Q 0(S)=-[(S-T) / 12],
$$

where $Q 0$ is the initial likelihood of stimulus $S$, and $T$ is the average threshold of the pilot subjects, which was -2.0 . Values for $S$ were between -8 and 10 logarithmic units.
Termination of testing was based on the likelihood ratio test proposed by Watson and Pelli (1983). In the present experiment, a 2 logarithmic-unit distance was selected as the width of the confidence interval, and the stopping criterion was a difference of $4.0 \mathrm{log}$ likelihood units between the stimulus with maximum likelihood and stimuli 2 logarithmic units away. Data from several pilot subjects indicated that this criterion produced a run length comparable to the 50 trials used in the Best PEST. If the stopping criterion was not met by 144 trials, the procedure was terminated and the subject was excluded from the analysis.

The Taylor and Creelman (1967) PEST procedure was implemented to run until the step size decreased to 1 logarithmic unit. Pilot data suggested that this would require $\mathbf{5 0}$ trials for the typical subject.

\section{Results and Discussion}

A total of 12 subjects failed to reach the stopping criterion ( 3 failed on both procedures, 2 failed only on the PEST, and 7 failed only on the QUEST), and 1 additional subject was eliminated due to experimental error, leaving 39 subjects for the analysis.

Table 2 presents the results expressed as the logarithmic stimulus-mask durations of Equation 5. There was no systematic difference between the procedures $[F(2,36)$ $=1.00$ ], nor were there differences between the three counterbalanced groups $[F(2,36)=1.70]$. There was a significant session effect, with the first session's threshold higher than later ones $[F(2,26)=8.35, p<.01]$.

The QUEST procedure ran more trials than the other two procedures. Apparently the confidence level selected for the stopping rule was too stringent or the slope parameter was underestimated.

The results replicate Shelton et al.'s (1982) conclusion that the original PEST and the Best PEST produce similar threshold estimates. This equivalence is extended to the QUEST procedure as well, and to situations involving inexperienced subjects and visual stimuli.

\section{CONCLUSIONS AND RECOMMENDATIONS}

The main result of the human-subjects experiment was that the three psychophysical procedures were indistinguishable in practice. Care was taken to estimate ac-

Table 2

Thresholds and Run Lengths for Human Subjects

\begin{tabular}{lcc}
\hline & $M$ & $S D$ \\
\hline \multicolumn{2}{c}{ Thresholds by Procedures } \\
Best PEST & .31 & 3.68 \\
PEST & .26 & 3.71 \\
QUEST & .74 & 3.19 \\
& Thresholds by Sessions & \\
First & 1.57 & 3.43 \\
Second & -.26 & 3.37 \\
Third & -.18 & 3.79 \\
& Trials Required & \\
Best PEST & 50.0 & 0 \\
PEST & 48.5 & 28.3 \\
QUEST & 62.9 & 24.1 \\
\hline
\end{tabular}

Note-Threshold data are stimulus-mask durations expressed in the logarithmic units of Equation 5. 
curately the slope parameters of the psychometric functions used in the maximum-likelihood procedures. The range of stimuli was relatively narrow, in deference to the Best PEST. The special requirements of the QUEST procedure were reasonably satisfied by appropriate stimulus scaling and accurate a priori information about the distribution of thresholds. These precautions lead us to conclude that the data were collected under conditions favorable to the maximum-likelihood methods. The small differences between the procedures in the computer simulations simply did not translate into measurable differences with human subjects.

Overall, we were impressed with the robustness of the original PEST procedure. Although computer simulations showed it to be somewhat less efficient than the maximumlikelihood methods, the differences do not seem to be relevant in actual use. The procedure makes no assumptions about the subject's psychometric function or the scaling of the stimuli. Our data suggest that it should be the procedure of choice for an investigator who is not in a position to worry about stimulus spacing, slope parameters, and a priori distributions. The PEST procedure can directly estimate any point on the psychometric curve, whereas the maximum-likelihood methods can find points other than the threshold parameter only by estimating the slope of the subject's psychometric function and calculating an offset from the threshold estimate as in Equation 4. The main disadvantage of PEST when used as a variable-trials procedure is that it can, under some conditions, take a seemingly interminable number of trials to converge. The stopping rule used in this study terminated testing when the step size was reduced to 1 logit unit, and this worked well. Shelton et al.'s (1982) data show that more stringent stopping rules, which required the procedure to run for more trials, were associated with greater variability in the threshold estimates than were less stringent stopping criteria. It is likely that subjects' performance suffers when the testing continues for long periods.

We are left with a number of impressions about the maximum-likelihood methods. The differences between simulated yes-no and 2AFC emphasize the importance of the psychophysical paradigm for the performance of the procedures. The advantages of maximum-likelihood methods in yes-no are reduced in 2AFC, apparently as a result of guessing-induced variability.

However, concerns that maximum-likelihood procedures are especially vulnerable to learning and lapses do not seem warranted, at least under the learning and lapsing assumptions explored here. The maximum-likelihood methods are roughly comparable to the PEST procedure in the 2AFC simulations.

QUEST is more stable than the Best PEST, and this is due to the use of an a priori distribution of threshold values to constrain the selection of test stimuli during the early trials. Users of the Best PEST in 2AFC should be wary of potential instability during early trials of a testing session, although restricting the range of test stimuli may control this problem. The slope parameter of the Best PEST should be chosen in 2AFC to be an overestimate. This is especially important in situations where lapsing may occur.

The QUEST confidence-interval approach to terminating testing is conceptually elegant, but has a number of complications in practice. The expected number of trials required to reach the stopping criterion is determined by the interaction among the width of the confidence inter$\mathrm{val}$, the level of confidence selected, and the slope of the subject's psychometric function. Under an unlucky combination of these variables, the QUEST procedure will not reach convergence. Furthermore, individual differences among subjects' psychometric functions affect the confidence level actually achieved by the QUEST procedure. For these reasons, we do not recommend QUEST with a confidence-interval stopping rule for $2 \mathrm{AFC}$. Simulations of QUEST running for a fixed number of trials produce results very close to those reported above for the confidence-interval approach. A fixed-trials approach also eliminates significant complications in implementing QUEST.

In fact, the major strength of the maximum-likelihood methods, in our view, is their use in fixed-trials procedures. This format can be especially convenient for the investigator and predictable for the subjects. Taylor and Creelman's (1967) PEST is our choice for a 2AFC procedure that runs a variable number of trials and stops when a performance criterion is reached.

We are tempted to suggest a hybrid maximumlikelihood procedure that would combine the most convenient and useful features of the two approaches investigated here. The hybrid method might select Pentland's (1980) fixed-trials procedure, with its less restrictive logistic function, coupled with Watson and Pelli's (1983) use of an a priori distribution of possible threshold values to stabilize the selection of test stimuli. Simulations of this procedure, shown in Figure 4, look promising. However, the human-subjects data do not lead us to believe that this procedure would be any better or worse in practice than the three approaches we tested. Psychophysical procedures have been actively refined over a century, and the result is a collection of methods that introduce error variances that are small compared with the uncontrolled error variances contributed by the subjects themselves. For this reason, small refinements in psychophysical methods are unlikely to result in measurable improvements in the quality of human $2 \mathrm{AFC}$ psychometric data.

\section{REFERENCES}

CoRnsweEt, T. N. (1962). The staircase-method in psychophysics. American Journal of Psychology, 75, 485-491.

Creelman, C. D., \& Kaplan, H. L. (1973). Simultaneous independent threshold estimates: Multiple PEST. Behavior Research Methods \& Instrumentation, 5, 89-92.

Emerson, P. L. (1984). Observations on a maximum likelihood method of sequential threshold estimation and a simplified approximation. Perception \& Psychophysics, 36, 199-203. 
EMERSON, P. L. (1986). Observations on maximum-likelihood and Bayesian methods of forced-choice sequential threshold estimation. Perception \& Psychophysics, 39, 151-153.

FinDLAY, J. M. (1978). Estimates on probability functions: A more virulent PEST. Perception \& Psychophysics, 23, 181-185.

GREEN, D. M. \& LUCE, R. D. (1975). Parallel psychometric functions from a set of independent detectors. Psychological Review, 82, 483-486.

Guilford, J. P. (1954). Psychometric methods. New York: McGraw-Hill.

HaLL, J. L. (1981). Hybrid adaptive procedure for estimation of psychometric functions. Joumal of the Acoustical Society of America, 69, $1763-1769$.

HALL, J. L. (1983). A procedure for detecting variability of psychophysical thresholds. Journal of the Acoustical Society of America, 73, 663-667.

Lieberman, H. R., \& Pentland, A. P. (1982). Microcomputer-based estimation of psychophysical thresholds: The Best PEST. Behavior Research Methods \& Instrumentation, 14, 21-25.

LEVITT, H. (1971). Transformed up-down methods in psychophysics. Journal of the Acoustical Society of America, 49, 467-477.

McKee, S. P., KleIN, S. A., \& Teller, D. Y. (1985). Statistical properties of forced-choice psychometric functions: Implications of probit analysis. Perception \& Psychophysics, 37, 286-298.

PentLAND, A. (1980). Maximum likelihood estimation: The best PEST. Perception \& Psychophysics, 28, 377-379.
Shelton, B. R., PiCardi, M. C., \& Green, D. M. (1982). Comparison of three adaptive psychophysical procedures. Journal of the Acoustical Society of America, 71, 1527-1533.

Shelton, B. R., SCARRow, I. (1984). Two-altemative versus threealternative procedures for threshold estimation. Perception \& Psychophysics, 35, 385-392.

SHIPLEY, E. F. (1961). Dependence of successive judgments in detection tasks: Correctness of the response. Journal of the Acoustical Society of America, 33, 1142-1143.

Taylor, M. M., \& CReelman, C. D. (1967). PEST: Efficient estimates on probability functions. Journal of the Acoustical Society of America, 41, 782-787.

Taylor, M. M., Forbes, S. M., \& Creelman, C. D. (1983). PEST reduces bias in forced choice psychophysics. Journal of the Acoustical Society of America, 74, 1367-1374.

WALD, A. (1947). Sequential analysis. New York: Wiley.

Watson, A. B., \& Pelli, D. G. (1983). A Bayesian adaptive psychometric method. Perception \& Psychophysics, 33, 113-120.

WETHERILL, G. B., \& LEVITT, H. (1965). Sequential estimation of points on a psychometric function. British Journal of Mathematical Statistical Psychology, 18, 1-10.

(Manuscript received October 14, 1986; revision accepted for publication March 16, 1987.) 